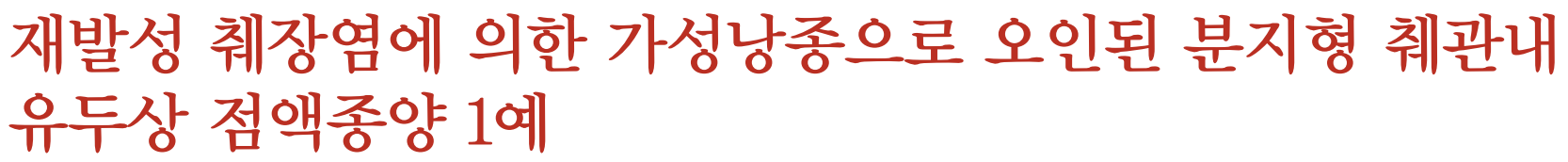

영남대학교 의과대학 내과학교실

강성훈 · 김국현 · 김태년

\title{
A Case of Branch Duct Intraductal Papillary Mucinous Neoplasm Mimicking Pseudocysts Complicated by Recurrent Pancreatitis
}

\author{
Sung Hoon Kang, Kook Hyun Kim, Tae Nyeun Kim \\ Division of Gastroenterology and Hepatology, Department of Internal Medicine, Yeungnam University College of Medicine, Daegu, Korea
}

\begin{abstract}
Branch duct intraductal papillary mucinous neoplasms (BD-IPMNs) are characterized by cystic dilation of secondary ducts clearly communicating with a normalsized main pancreatic duct and seem to have a less aggressive clinical course than those arising from the main duct. BD-IPMNs are related to pancreatitis but the causal relationship is unclear. We report a case of a 62-year-old woman initially thought to have pseudocysts complicated by idiopathic recurrent pancreatitis but was finally diagnosed with BD-IPMN leading to recurrent acute pancreatitis attacks. The patient had six episodes of acute pancreatitis over 5 years. An abdominal computed tomography scan revealed two cystic lesions of 2.0 and $1.5 \mathrm{~cm}$ in the pancreatic body, which appeared at the second episode of acute pancreatitis. Each pancreatitis episode improved with conservative treatment, but the cystic lesions increased in size to 2.5 and $3.5 \mathrm{~cm}$ during the late follow-up period. A distal pancreatectomy was performed under the diagnosis of recurrent pancreatitis caused by BD-IPMN. The pathological findings revealed BD-IPMN with moderate dysplasia. We herein present a case of BDIPMN mimicking pancreatic pseudocysts with a review of the literature.
\end{abstract}

Korean J Pancreatobiliary 2015;20(2):94-98

Keywords: Acute recurrent pancreatitis, Intraductal papillary mucinous neoplasm, Pancreas, Pseudocyst

\author{
Received Jan. 17, 2015 \\ Revised Mar. 24, 2015 \\ Accepted Mar. 31, 2015
}

Corresponding author: Tae Nyeun Kim Division of Gastroenterology and hepatology, Department of Internal Medicine, Yeungnam University College of Medicine, 170 Hyeonchungro, Nam-gu, Daegu 705-802, Korea

Tel. +82-53-620-3842 Fax. +82-53-654-8386

E-mail; tnkim@yu.ac.kr

This is an Open Access article distributed under the terms of the Creative Commons Attribution Non-Commercial License (http:// creativecommons.org/licenses/by-nc/3.0/) which permits unrestricted non-commercial use, distribution, and reproduction in any medium, provided the original work is properly cited.

Copyright $\odot 2015$ by The Korean Journal of Pancreas and Biliary Tract

\section{INTRODUCTION}

Intraductal papillary mucinous neoplasm (IPMN) of the pancreas is a cystic neoplasm originating from the epithelium of the pancreatic duct and is characterized by papillary intraductal growth of mucin-producing cells with variable degrees of cytological atypia, mucin hypersecretion, and cystic dilation of the involved pancreatic ducts. ${ }^{1,2}$ IPMNs are classified as the main duct type, branch duct type, or mixed type according to the area of the involved ducts. The main duct type of IPMNs (MD-IPMNs) are frequently associated with a pancreatic duct obstruction and pancreatitis. Previous studies have reported an incidence of acute or chronic pancreatitis associated with MD-IPMNs ranging from 14 to 
53\%. ${ }^{3-5}$ However, branch duct type of IPMNs (BD-IPMNs), which are usually small and indolent in their clinical course, are rarely associated with obstructive pancreatitis, and the incidence of acute or chronic pancreatitis is $5 \% .{ }^{6}$ Furthermore, BD-IPMNs are frequently found incidentally during routine radiological examinations and can be misdiagnosed as pseudocysts or other kinds of cystic tumors because of nonspecific imaging findings.

Although BD-IPMNs can also be the cause of acute or recurrent pancreatitis, ${ }^{7,8}$ the causal relationship is unclear, and published case reports are rare. Herein, we report a case of BD-IPMN leading to recurrent attacks of acute pancreatitis, which was initially thought to be pseudocysts complicated by idiopathic recurrent pancreatitis.

\section{CASE}

A 62-year-old woman was admitted to the hospital due to acute abdominal pain. The patient had suffered six episodes of recurrent acute pancreatitis during the past 5 years. She had been taking medications for diabetes mellitus, hypertension, and hypothyroidism. She did not smoke or drink alcohol.

The first acute pancreatitis episode occurred 5 years ago. Laboratory findings at that time included leukocyte count

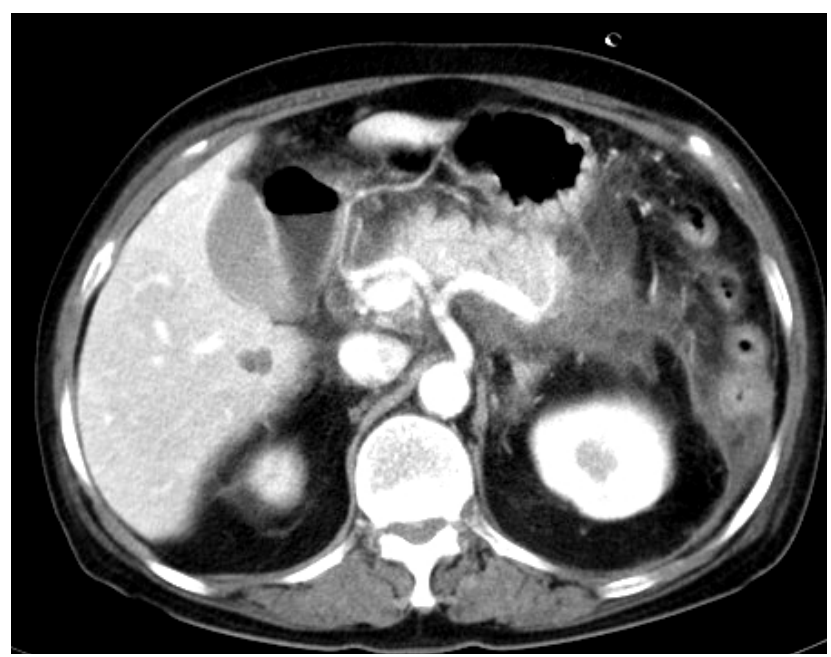

Fig. 1. Abdominal computed tomography at first visit. Diffuse pancreatic swelling with severe peripancreatic fat infiltration with fluid collection was noted.
$19,700 / \mathrm{mm}^{3}$, hemoglobin $11.6 \mathrm{gm} / \mathrm{dL}$, platelets $386,000 /$ $\mathrm{mm}^{3}$, serum amylase $4,100 \mathrm{U} / \mathrm{L}$, serum lipase $3,800 \mathrm{U} / \mathrm{L}$, total protein $6.18 \mathrm{~g} / \mathrm{dL}$, albumin $3.85 \mathrm{~g} / \mathrm{dL}$, total bilirubin 0.7 $\mathrm{mg} / \mathrm{dL}$, aspartate transaminase $12 \mathrm{U} / \mathrm{L}$, alanine transaminase $14 \mathrm{U} / \mathrm{L}$, alkaline phosphatase $153 \mathrm{U} / \mathrm{L}$, gamma glutamytranspeptidase $14 \mathrm{U} / \mathrm{L}$, total cholesterol $150 \mathrm{mg} / \mathrm{dL}$, triglycerides $100 \mathrm{mg} / \mathrm{dL}$, calcium $7.0 \mathrm{mg} / \mathrm{dL}$, and inorganic phosphorus $2.8 \mathrm{mg} / \mathrm{dL}$. An abdominal computed tomography (CT) scan showed severe peripancreatic fat infiltration with fluid collection and pleural effusion (Fig. 1). Neither a cystic lesion nor ductal dilation was found at this time. No gallstones were detected on an ultrasonogram. A diagnosis of idiopathic pancreatitis was made. She improved with conservative treatment and was administered ursodeoxycolic acids under suspicion of microlithiasis as the cause of the pancreatitis.

She was readmitted 17 months later due to severe epigastric pain with elevated levels of serum amylase and lipase. An abdominal CT scan showed peripancreatic fat infiltration and two cystic lesions $(2.0$ and $1.5 \mathrm{~cm})$ in the pancreatic body, which did not seem to communicate with the pancreatic duct (Fig. 2). The cystic lesions were considered pseudocysts because of the low-attenuation, unilocular cyst with accompanying acute pancreatitis. Her symptoms improved with conservative treatment. Two additional recurrent pan-

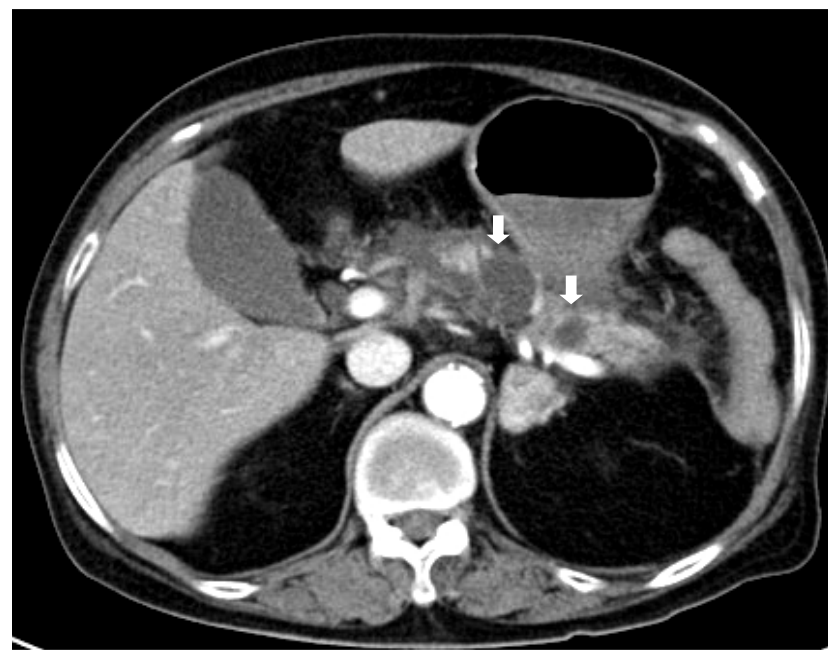

Fig. 2. Abdominal computed tomography at second attack of acute patncreatitis. Peipancreatic fat infiltration and about $2 \mathrm{~cm}$ and $1.5 \mathrm{~cm}$ sized cystic lesions with thin wall in the body of the pancreas were seen. 
creatitis episodes developed 34 and 52 months after the first admission, but her symptoms improved with conservative treatment. The size of pancreatic cystic lesions decreased initially after the third pancreatitis episode but remained unchanged during further follow-up. A tubular shaped or grape-like cystic lesion without changes in size and shape in follow-up images even after full recovery of acute recurrent pancreatitis is highly suggestive of BD-IPMN. The abdominal CT scan revealed no typical BD-IPMN findings. However, we were strongly suspicious of BD-IPMN as the cause for the acute recurrent pancreatitis because of the lack of a change in the size and shape of the cystic lesions during the follow-up. Therefore, surgical resection or further evaluation, such as magnetic resonance cholangiopancreatography, endoscopic ultrasonography, and 18F-fluorodeoxyglucose-positron emission tomography combined with CT, were strongly recommended. However, the patient refused both surgical resection and further evaluation.

The patient was readmitted 4 months later due to a recurrent attack of acute pancreatitis. The cystic lesions had increased in size to 2.5 and $3.5 \mathrm{~cm}$ (Fig. 3). A distal pancreatectomy was performed after the pancreatitis improved under a recurrent pancreatitis diagnosis due to BD-IPMN. The gross findings of the resected specimen showed multiloculated cysts with septation (Fig. 4). The histological find-

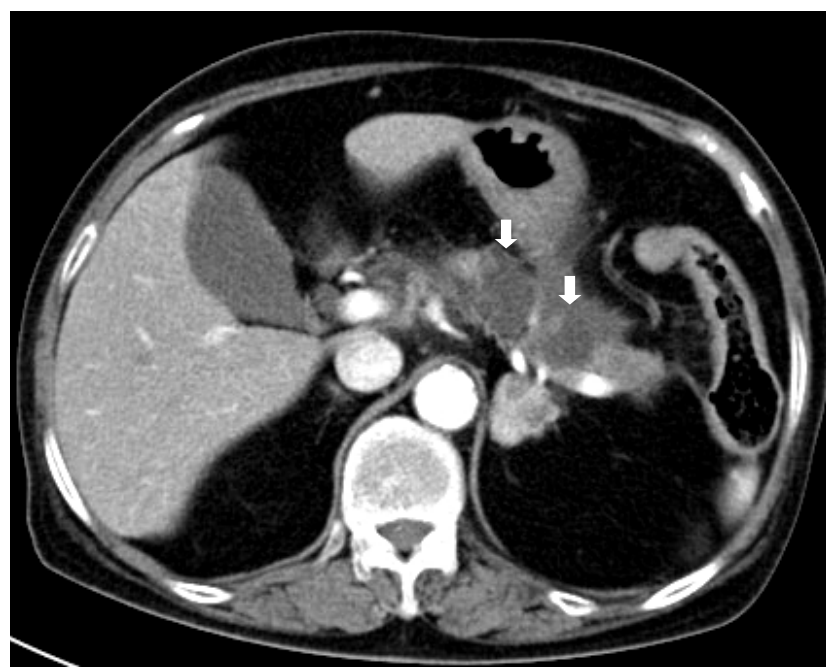

Fig. 3. Abdominal computed tomography findings at last attack of acute pancreatitis. The cystic lesions increased in sized up to $2.5 \mathrm{~cm}$ and $3.5 \mathrm{~cm}$. ings revealed two cystic lesions of $2.0 \times 1.5 \mathrm{~cm}$ and $3.3 \times 2.3$ $\mathrm{cm}$ with multilobulation, and they were connected to each other in the pancreatic body (Fig. 5A). The cystic lesions were filled with mucin in the cytoplasm and had scattered elongated nuclei with moderate focal dysplasia (Fig. 5B). After surgery, the patient was discharged without complications and no recurrence of acute pancreatitis has been reported during the follow-up.

\section{DISCUSSION}

IPMN of the pancreas is a rare pancreatic tumor originating from the epithelium of the pancreatic duct that is characterized by mucin production. In most IPMN cases, hypersecretion of mucin leads to cystic dilatation of the involved ducts. IPMNs are categorized into the main duct type, the branch duct type, and the mixed type based on imaging studies or histology. The majority of the BD-IPMNs are small cystic masses without dilation of the main duct, whereas MD-IPMNs are highly suggested when dilatation of main pancreatic duct is $>1 \mathrm{~cm}$.

MD-IPMNs and BD-IPMNs have significant differences in their clinical course and malignant potential. BD-IPMNs are typically smaller, less complex (less papillary), and less malignant compared to those of MD-IPMNs, which ex-

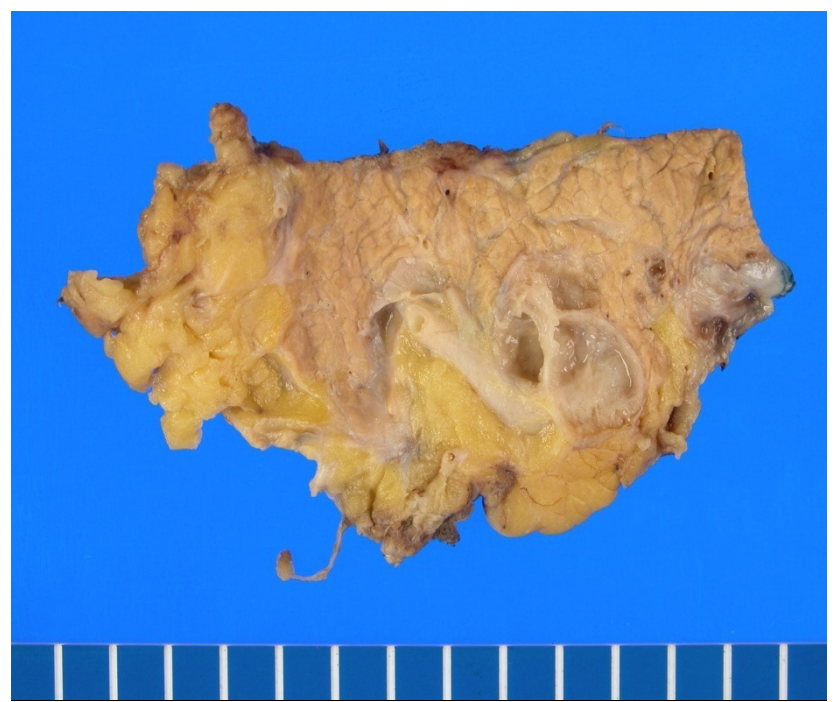

Fig. 4. Gross findings of the resected specimen. At distal pancreas, multiloculated cysts with septation were noted. 

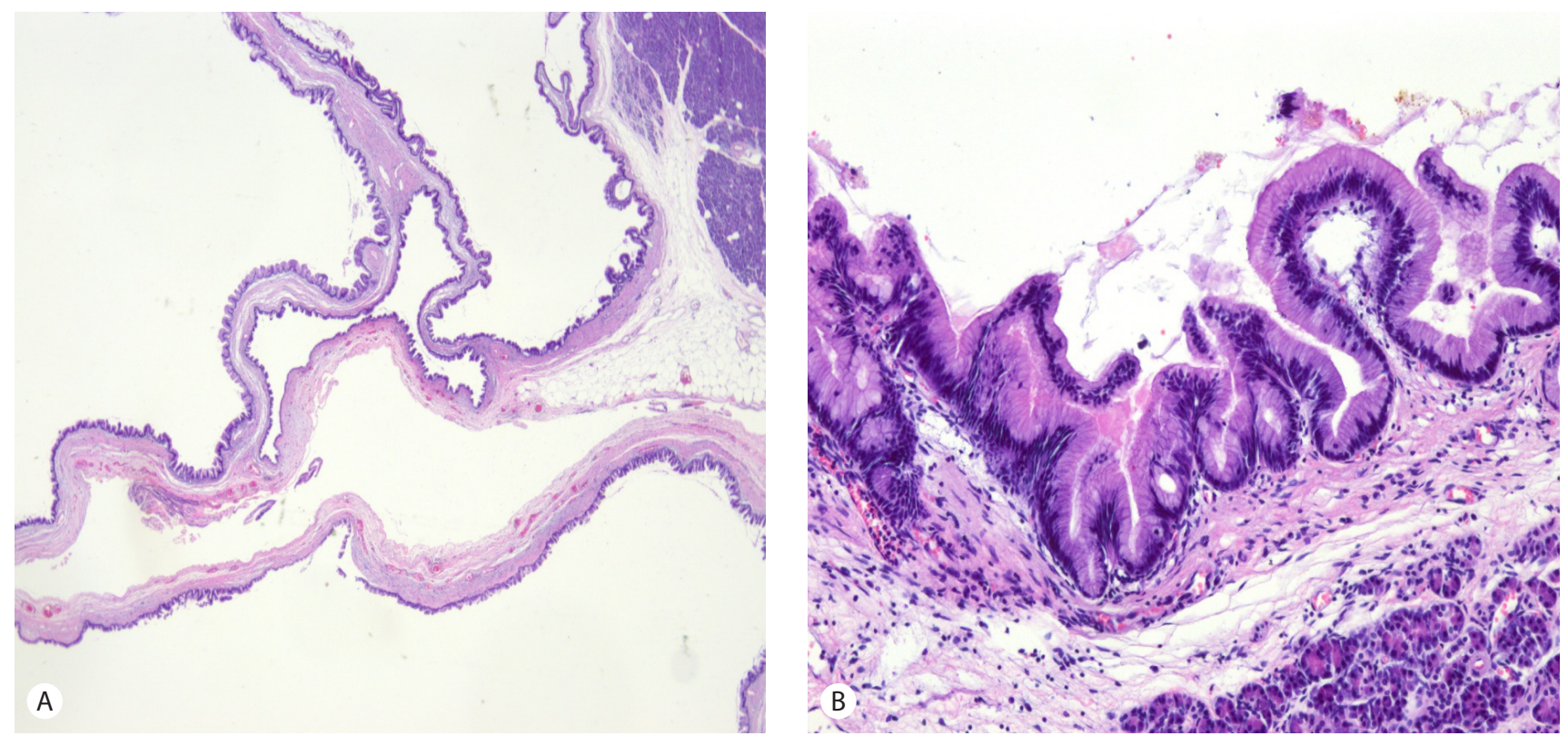

Fig. 5. Histologic findings of the resected specimen. (A) About $2.0 \times 1.5 \mathrm{~cm}$ and $3.3 \times 2.3 \mathrm{~cm}$ sized 2 cystic lesions with multilobulation were noted. The cystic lesions were connected each other $(H \& E, \times 40)$. (B) The lining of the cyst showed papillary structure with focal moderate dysplasia (H\&E, $\times 200)$.

plains why many BD-IPMNs have been successfully managed conservatively. The reported incidence rate of cancer in the main and branch ducts of IPMNs are $57-92 \%$ and $6-46 \%$, respectively. ${ }^{9,10} \mathrm{BD}-\mathrm{IPMN}$ are asymptomatic in $86 \%$ of cases, and abdominal pain is the most frequent symptom in symptomatic cases. The mechanism of pancreatitis in BD-IPMNs seems to be similar to that of MDIPMNs. Viscous mucinous secretions from IPMNs may obstruct the main pancreatic duct or branch duct, leading to elevated pancreatic duct pressure and subsequent pancreatitis. $^{11}$

Concomitant BD-IPMNs associated with acute pancreatitis are frequently confused with pseudocysts. One case report on IPMNs presented that the initial diagnosis was a pseudocyst, and a pseudocyst-jejunal anastomosis was performed. ${ }^{12}$ In our case, we initially considered that the cystic lesions were pseudocysts complicated by acute pancreatitis because the lesions were not present at the time of the first attack but appeared after the second attack of pancreatitis. The relatively long interval of $>1$ year between the first and second pancreatitis attacks made the diagnosis more difficult. We thought that the initial pancreatitis attack may have been caused by pre-existing small BD-IPMNs, which were masked on the CT scan, by severe inflammation during the first attack of pancreatitis. Clues leading to the diagnosis of BD-IPMN in our case were as follows: (1) no obvious potential cause for recurrent pancreatitis; (2) the relatively small cystic lesions did not disappear even after improvement in pancreatitis; and (3) the size and position of the cysts were unchanged during a long-term follow-up. Some fluctuations in the size of the BD-IPMNs were likely related with pancreatitis, which can temporarily increase the size of BD-IPMNs by blocking the pancreatic duct with mucin plugs.

IPMNs are generally slowly growing tumors with a good prognosis. A recent international consensus guideline recommended nonsurgical management with close observation of IPMNs that arise from branch ducts and that fail to meet certain "high-risk" criteria, including causes attributable to the cyst (such as pancreatitis), cyst size of at least $30 \mathrm{~mm}$, presence of intramural nodules, or cyst fluid cytology suspicious or positive for malignancy. ${ }^{13}$ BD-IPMNs appear to be a risk factor for pancreatic cancer and careful observation is recommended. ${ }^{14,15}$ Although the BD-IPMN in our case was a very slow growing tumor over 5 years, there was a high possibility of progression to malignancy if surgical resection 
was not performed because the pathological findings showed moderate dysplasia.

In conclusion, BD-IPMNs should be considered as a cause of recurrent pancreatitis of unknown etiology. The presence of pancreatic cystic lesions in patients with idiopathic pancreatitis suggests the possibility of BD-IPMNs as the cause of the pancreatitis, particularly when the cysts are small and unchanged during follow-up.

국문 색인: 재발성 급성 췌장염, 췌관내 유두상 점액종양, 췌장, 가성낭종

\section{Conflicts of Interest}

The author has no conflicts to disclose.

\section{REFERENCES}

1. Rivera JA, Fernandez-del Castillo C, Pins M, et al. Pancreatic mucinous ductal ectasia and intraductal papillary neoplasms. Ann Surg 1997;225:637-646.

2. Traverso LW, Peralta EA, Ryan JA Jr, Kozarek RA. Intraductal neoplasms of the pancreas. Am J Surg 1998;175:426-432.

3. Salvia R, Bassi C, Falconi M, et al. Intraductal papillary mucinous tumors of the pancreas. Surgical treatment: at what point should we stop? JOP. J Pancreas 2005;6:112-117.

4. Loftus EV Jr, Olivares Pakzad BA, Batts KP, et al. Intraductal papillary mucinous tumors of the pancreas: clinicopathologic features, outcome, and nomenclature: members of the pancreas clinic, and pancreatic surgeons of Mayo clinic. Gastroenterology 1996;110:19091918.

5. Zamora C, Sahel J, Cantu DG, et al. Intraductal papillary or mucinous tumors (IPMT) of the pancreas: report of a case series and review of the literature. Am J Gastroenterol 2001;96:1441-1447.

6. Jang JW, Kim MH, Jeong SU, et al. Clinical characteristics of intraductal papillary mucinous neoplasm manifesting as acute pancreatitis or acute recurrent pancreatitis. J Gastroenterol Hepatol 2013;28:731738.

7. Chung JP, Chi SW, Park YN, et al. A case of minute intraductal papillary mucinous tumor of the pancreas presenting with recurrent acute pancreatitis. Yonsei Medical Journal 2000;41:528-532.

8. Ringold DA, Shorff P, Sikka SK, et al. Pancreatitis is frequent among patients with side-branch intraductal papillary mucinous neoplasia diagnosed by EUS. Gastrointest Endosc 2009;70:488-494.

9. Sugiyama $M$, Izumisato $Y$, Abe $N$, et al. Predictive factors for malignancy in intraductal papillary-mucinous tumours of the pancreas. Br J Surg 2003;90:1244-1249.

10. Sohn TA, Yeo CJ, Cameron JL, et al. Intraductal papillary mucinous neoplasms of the pancreas: an updated experience. Ann Surg 2004;239:788-797.

11. Hata T, Sakata N, Okada T, et al. Dilated papilla with mucin extrusion is a potential predictor of acute pancreatitis associated with intraductal papillary mucinous neoplasms of pancreas. Pancreatology 2013;13:615-620

12. Abu-Hilal M, Salvia R, Casaril A, et al. Obstructive chronic pancreatitis and/or intraductal papillary mucinous neoplasms (IPMNs): a 21year long case report. JOP 2006;7:218-221.

13. Tanaka M, Chari S, Adsay V, et al. International consensus guidelines for management of intraductal papillary mucinous neoplasms and mucinous cystic neoplasms of the pancreas. Pancreatology 2006;6:1732.

14. Rodriguez JR, Salvia R, Crippa S, et al. Branch-duct intraductal papillary mucinous neoplasms: observations in 145 patients who underwent resection. Gastroenterology 2007;133:72-79.

15. Salvia R, Crippa S, Falconi M, et al. Branch-duct intraductal papillary mucinous neoplasms of the pancreas: to operate or not to operate? Gut 2007;56:1086-1090. 